\title{
Evaluación de la terapia mecánica periodontal en bolsas profundas: Respuesta clínica y bacteriológica
}

\section{Evaluation of periodontal mechanical therapy in deep pockets: Clinical and bacteriological response}

\author{
Bazzano $\mathrm{G}^{1}$, Parodi $\mathrm{R}^{2}$, Tabares $\mathrm{S}^{3}$, Sembaj $\mathrm{A}^{4}$
}

\begin{abstract}
RESUMEN
Objetivos: Evaluar la composición microbiológica y los parámetros clínicos de bolsas periodontales $\geq 5 \mathrm{~mm}$ de profundidad al inicio, $1 \mathrm{semana,} 3$ y 12 meses post raspado y alisado radicular. Materiales y Métodos: Se tomaron registros clínicos y muestras de placa subgingival de 44 sitios de pacientes con diagnóstico de periodontitis crónica. Se identificaron por técnica de Reacción en Cadena de la Polimerasa (PCR) patógenos putativos periodontales: Aggregatibacter actinomycetemcomitans (Aa), Porphyromonas gingivalis (Pg), Treponema denticola (Td), Tannerella forsythia (Tf) y Prevotella intermedia (Pi). Los pacientes recibieron terapia mecánica periodontal y fueron reevaluados a los 7 días, 3 y 12 meses. Resultados: Luego del tratamiento, todos los parámetros clínicos (Placa Bacteriana, Hemorragia, Supuración, Profundidad al Sondaje y Nivel de Inserción Clínica) se redujeron significativamente y los valores obtenidos se mantuvieron hasta los 12 meses. Al inicio, las especies bacterianas prevalentes fueron $P g$, presente en $66 \%$ de los sitios, $T f(55 \%)$ y $T d(41 \%)$. Los sitios más profundos se relacionaron con las asociaciones $T f-T d$ $(6.8 \mathrm{~mm})$ y $T f-T d-P i(7 \mathrm{~mm})$. Post terapia, el número de sitios positivos para $T d, T f$ y $P g$ se redujo significativamente. Conclusiones: El raspado y alisado radicular mejoró significativamente los parámetros clínicos y redujo la prevalencia de los patógenos periodontales $P g, T f$ y $T d$ en bolsas periodontales profundas. Los resultados obtenidos se mantuvieron hasta los 12 meses. No se detectaron mayores pérdidas de inserción clínica en el $86 \%$ de los sitios a 3 meses y en $79 \%$ a los 12 meses. Los sitios en los que el tratamiento no fue efectivo en la eliminación de patógenos a los 12 meses desarrollaron mayores profundidades de sondaje.
\end{abstract}

Rev. Clin. Periodoncia Implantol. Rehabil. Oral Vol. 5(3); 123-128, 2012.

Palabras clave: Raspado y alisado radicular, bacteriología, periodontitis.

\section{ABSTRACT}

Objectives: To evaluate the microbial composition and clinical parameters of periodontal pockets with probing depth $\geq 5 \mathrm{~mm}$ at baseline, 1 week, 3 and 12 months after scaling and root planning. Methods: Clinical parameters were measured and bacterial samples were collected from 44 sites in 11 patients with chronic periodontitis. By means of Polymerase Chain Reaction (PCR) the presence of Aggregatibacter actinomycetemcomitans (Aa), Porphyromonas gingivalis $(P g)$, Treponema denticola $(T d)$, Tannerella forsythia $(T f)$ and Prevotella intermedia $(P i)$ was estimated. The patients received mechanical periodontal therapy and were evaluated after 1 week, 3 months and 12 months. Results: After treatment, all clinical parameters (Plaque, Bleeding on Probing, Supuration, Probing Pocket Depth and Clinical Attachment Level) were significantly reduced, and the values obtained were maintained up to the 12 months that the study lasts. At baseline, the most prevalent species were $P g$, present in $66 \%$ of the sites, $\mathrm{Tf}(55 \%)$ and $T d(41 \%)$. The deepest sites were related to the association $T f-T d(6.8 \mathrm{~mm})$ and $T f-T d-P i(7 \mathrm{~mm})$. The number of positive sites for $T d$, $T f$ and $\mathrm{Pg}$ was significantly reduced after therapy. Conclusions: Scaling and root planning improve significantly clinical parameters as well as reduce the prevalence of periodontal pathogens $P g, T d$ and $T f$ in deep periodontal pockets. The results obtained were maintained up to 12 months. No further clinical attachment loss was found in $86 \%$ of the sites at 3 months and $79 \%$ at 12 months. The sites where the treatment failed in removing pathogens developed at 12 months greater probing pocket depths.

Rev. Clin. Periodoncia Implantol. Rehabil. Oral Vol. 5(3); 123-127, 2012.

Key words: Non-surgical periodontal therapy, bacteriology, periodontitis.

\section{INTRODUCCIÓN}

Las periodontitis son infecciones multifactoriales iniciadas por complejos bacterianos que interactúan con los tejidos y células del hospedero provocando una respuesta inmunoinflamatoria, que conduce a la destrucción de los tejidos de soporte de las piezas dentarias, incluyendo el ligamento periodontal y el hueso alveolar. La forma más frecuente es la periodontitis crónica, que se manifiesta con inflamación de los tejidos de soporte del diente y pérdida progresiva del ligamento periodontal y hueso alveolar ${ }^{(1-3)}$.
El factor etiológico primario de las periodontitis es el complejo biofilm microbiano que coloniza la región del surco entre la superficie dentaria y el margen gingival(4). La composición microbiana del biofilm puede variar entre diferentes poblaciones y aún entre individuos con similares características clínicas y demográficas ${ }^{(5-9)}$. En un trabajo previo $^{(10)}$, observamos una elevada prevalencia de $P g$, y baja frecuencia de sitios positivos para $\mathrm{Pi}$ y $\mathrm{Aa}$, si se compara con lo descrito por otros autores ${ }^{(11-13)}$.

La terapia mecánica no quirúrgica es el tratamiento de la inflamación gingival y periodontal a través de la remoción mecánica de

1. Doctora en Odontología. Cátedra de Periodoncia, Facultad de Odontología. Universidad de Buenos Aires. Argentina.

2. Doctor en Odontología. Facultad de Odontología, Universidad Nacional de Córdoba. Argentina.

3. Técnica de Laboratorio Clínico e Histopatológico. Facultad de Ciencias Médicas, Universidad Nacional de Córdoba. Argentina.

4. Doctora en Ciencias Biológicas. Facultad de Ciencias Médicas, Universidad Nacional de Córdoba. Argentina. 
irritantes de las superficies dentarias, técnica que se repite hasta que los tejidos blandos adyacentes recuperen un estado saludable ${ }^{(14)}$.

El procedimiento incluye control de placa, raspado y alisado radicular y es uno de los más utilizados para el tratamiento de las enfermedades periodontales ${ }^{(15)}$. Sus efectos clínicos han sido bien documentados. La mayoría de los autores coinciden en que el tratamiento mecánico es efectivo en la reducción de la inflamación, disminución de la profundidad de sondaje y pérdida de inserción clínica ${ }^{(14,16-19)}$. Sin embargo, pocos o ninguno demuestran si esas variaciones representan parámetros de éxito clínico en cuanto a recuperación de un estado periodontal compatible con salud.

El éxito del tratamiento estaría asociado a la reducción de patógenos periodontales del área subgingival y si bien se han observado profundos cambios en la composición de la microflora, estos serían transitorios, especialmente en los sitios profundos ${ }^{(15,20-25)}$.

Entre los métodos de diagnóstico bacteriológico disponibles en la actualidad, que han permitido identificar especies que eran raras o no detectables por métodos de cultivo, se cuenta con la Reacción en Cadena de la Polimerasa $(\mathrm{PCR})^{(26)}$, una técnica rápida, precisa y sensible para la detección de secuencias especificas de DNA bacteriano en una muestra ${ }^{(27)}$

El presente trabajo se realizó con el fin de: (i) Determinar la prevalencia de $P g, T f, T d, A a$ y $P i$ en el biofilm subgingival de pacientes con periodontitis crónica severa antes y después del tratamiento periodontal; (ii) Evaluar la efectividad de la terapia mecánica periodontal en la eliminación de patógenos y en los parámetros clínicos en bolsas profundas a 12 meses post tratamiento; y (iii) Reconocer si la mejoría clínica representa éxito terapéutico de acuerdo a criterios de evaluación conocidos.

\section{MATERIAL Y MÉTODO}

\section{Población y Muestra}

El estudio fue diseñado conforme a los requerimientos de la Declaración de Helsinki, fue aprobado por el Comité de Ética de la Facultad de Odontología de la Universidad Nacional de Córdoba (UNC). Aproximadamente 100 pacientes que concurrieron a las Cátedras de Periodoncia de la Facultad de Odontología de la UNC y de la Universidad de Buenos Aires fueron invitados a participar en el estudio. Teniendo en cuenta los criterios de inclusión de estar sistémicamente sanos, con al menos una pieza dentaria unirradicular por cuadrante con Profundidad de Sondaje $\geq 5 \mathrm{~mm}$ y habiendo excluido fumadores, embarazadas, los que hubieran recibido tratamiento periodontal en los últimos 2 años o medicación antibiótica en los últimos 6 meses; quedaron veinte pacientes con diagnóstico clínico radiográfico de Periodontitis Crónica. El criterio de diagnóstico de Periodontitis Crónica incluye inflamación de los tejidos de soporte acompañada por pérdida de inserción y pérdida ósea(1). Estos pacientes fueron informados de la naturaleza del estudio y dieron su consentimiento por escrito. De cada paciente se seleccionaron para ser evaluados 4 sitios con PS $\geq 5 \mathrm{~mm}$ de las caras proximales de piezas unirradiculares, uno por cuadrante. A lo largo del estudio, 9 pacientes fueron excluidos por tomar medicación o por no concurrir a las citas de evaluación. Completaron el estudio 11 pacientes $(6$ mujeres y 5 hombres).

\section{Evaluación Clínica}

Se registraron: Presencia de placa bacteriana supragingival (PB), Hemorragia al sondaje (H), Supuración (S), y se midieron, utilizando sonda tipo Marquis (Hu-Friedy): Profundidad al Sondaje (PS) y Nivel de Inserción Clínica (NIC). Se tomaron radiografías periapicales con técnica de cono largo. Todas las evaluaciones fueron realizadas por el mismo examinador $(\mathrm{GB})$.

\section{Evaluación Bacteriológica}

Las muestras para el análisis microbiológico se tomaron luego de la eliminación de la placa supragingival y el aislamiento del sitio seleccionado con gasa estéril. Se introdujeron consecutivamente 5 conos de papel estériles mediante movimientos de cateterismo en cada uno de los sitios seleccionados y permanecieron en la profundidad de la bolsa periodontal durante 60 segundos cada uno. Los conos se colocaron en tubos tipo Eppendorf estériles y la muestra se conservó a $4^{\circ} \mathrm{C}$ hasta su procesamiento.

EI DNA se extrajo de las muestras por técnicas convencionales y se amplificó, mediante PCR una secuencia específica y altamente conservada del gen 16S rRNA de 960 pb para identificar bacterias Gram negativas. Una vez confirmada la presencia de DNA bacteriano Gram negativo, en la misma muestra se realizaron amplificaciones con oligonucleótidos específicos para amplificar secuencias genómicas de: $P g, P i, T d, T f$ y $A a$.

Los productos de PCR se visualizaron en geles de agarosa al $1.6 \%$ teñidos con bromuro de etidio y se fotografiaron bajo luz ultravioleta. Un marcador de tamaño molecular se utilizó como referencia del tamaño del producto de amplificación (Perkin Elmers 100 bp).

\section{Tratamiento}

Una vez firmados los consentimientos informados y realizados los registros clínicos y radiográficos y tomadas las muestras para el análisis bacteriológico, se inició el tratamiento propuesto de terapia relacionada con la causa.

El mismo consistió en información al paciente sobre la naturaleza de la enfermedad, su pronóstico y plan de tratamiento, además de la instrucción en técnicas de higiene oral. Se indicaron los elementos para la higiene de caras libres y proximales que se consideraron más adecuados para cada caso en particular. El objetivo de esta fase fue lograr el compromiso del paciente con el tratamiento y la adquisición de destreza para el control personal del biofilm supragingival.

A continuación, se procedió a las maniobras de raspado y alisado radicular bajo efecto de anestesia local. Para esto se utilizó juego reducido de curetas Gracey $(7 / 8,11 / 12,13 / 14)$ (Hu-Friedy $\left.{ }^{\circledR}\right)$ y cavitador sónico neumático (KMD $®)$. La evaluación inmediata de la efectividad del raspado y alisado radicular se realizó por medios visuales y táctiles utilizando sonda periodontal tipo Marquis (Hu-Friedy ${ }^{\circledR}$ ).

\section{Fase Post Tratamiento}

Después de 1 semana de realizado el tratamiento, se registraron nuevamente los indicadores clínicos de PB, H, S, PS y NIC Se tomaron nuevas muestras para análisis bacteriológico y se reforzó la motivación para el control de placa supragingival.

La toma de registros clínicos y bacteriológicos conjuntamente con el refuerzo de las técnicas de higiene oral se repitieron a los 3 y 12 meses de realizada la terapia causal.

\section{Evaluación del Resultado al Tratamiento}

Según la American Academy of Periodontology ${ }^{(28)}$, los signos más aceptados como señal de gozar de buena salud periodontal incluyen la ausencia de signos inflamatorios como rubor, hinchazón, presencia de exudado y sangrado al mínimo sondaje y mantenimiento de una adherencia epitelial funcional. Sin embargo, la valoración de los resultados del tratamiento periodontal depende en gran medida del criterio de evaluación elegido.

Para este trabajo se utilizó una adaptación del sistema creado por Lundgren y cols. ${ }^{(29)}$. El mismo comprende 3 niveles diferentes que describen un estándar de salud deseable para un periodonto remanente tratado por periodontitis. Los criterios aplicados se describen en la Tabla 1 y representan los parámetros clínicos de evaluación objetiva para el mantenimiento de "perfectas" condiciones clínicas del periodonto remanente luego del tratamiento de periodontitis

\section{Análisis Estadístico}

A fin de validar los cambios en los parámetros clínicos PS y NIC se utilizó la prueba ANOVA para medidas repetidas. El test de Friedman se utilizó para evaluar los cambios en PB, $\mathrm{H}$ y S como respuesta al tratamiento. El mismo se aplicó también para establecer la significación en los cambios en la composición bacteriana de los sitios estudiados. Para datos numéricos se utilizó el test t de Student para datos apareados. Para evaluar la significación de los cambios en los distintos momentos del estudio (diseño antes y después) se utilizaron las pruebas Mc Nemar y $Q$ de Cochran. Para todas las pruebas se consideró un valor $p \leq 0.05$ para la significación. Se trabajó con un nivel de significación de $\alpha=0.05$. El software utilizado fue el SPSS Statistics versión 17. 


\section{RESULTADOS}

Inicialmente formaron parte del estudio 20 pacientes, de ellos, 11 lo concluyeron, 6 mujeres y 5 hombres. Su edad promedio fue $48.81 \pm 5.75$ años (38-55 años). Se analizaron 44 sitios.

\section{Resultados Clínicos}

Los datos clínicos de los sitios analizados correspondientes a las diferentes fases del tratamiento se muestran en la Tabla 2. Luego del tratamiento, se obtuvo una significativa reducción en la presencia de PB y $\mathrm{H}$ al sondaje, presentes al inicio en el $100 \%$ de los sitios, como así también en S. Los valores medios de PS y NIC disminuyeron significativamente y se observó que los resultados obtenidos a los 3 meses se mantuvieron estables hasta los 12 meses que duró el estudio.

\section{Resultados Bacteriológicos}

La frecuencia de las cinco bacterias estudiadas se describe en la Tabla 3. Al inicio del estudio, en el $86.4 \%$ de los sitios se identificaron por PCR una o más de las especies bacterianas, mientras que el $5 \%$ de los sitios estudiados contenía otras especies Gram (-). Pg es la especie prevalente, identificada en el $66 \%$ de los sitios, seguida por $T f$ con un $54.54 \%$ y $T d$ con $40.9 \%$. En esta etapa del estudio, las especies identificadas como menos frecuentes fueron $\mathrm{Aa}$ y $\mathrm{Pi}$ en 9 y $6.8 \%$ de los sitios, respectivamente y sólo asociadas a alguna/s de las otras especies anteriormente mencionadas.

La prevalencia de $P g, T d$ y $T f$ disminuyó significativamente luego del tratamiento, obteniéndose los mejores resultados a los 3 meses. Los valores obtenidos para $P g$ y $T f$ se mantuvieron estables hasta los 12 meses. Mientras que una repoblación por parte de $T d$ se pudo observar en este control, aunque sin alcanzar los valores iniciales. Se analizaron las asociaciones entre diferentes especies y se observó que el $41 \%$ de los sitios mostró dos especies bacterianas, siendo la asociación $\mathrm{Pg}$-Tf la más frecuente. Se observaron combinaciones de hasta cuatro especies.

En la Tabla 4 se muestra la relación entre la composición microbiana y la PS media en cada etapa del tratamiento. Observamos que las medidas más profundas de PS se vinculan con la asociación de $T f-T d$ y $T f-T d-P i$

La prevalencia de $P g, T d$ y $T f$ disminuyó significativamente luego del tratamiento, cuantificando medidas de PS de mejora periodontal a los tres meses post-tratamiento. Estos valores de PS asociados a la presencia de $\mathrm{Pg}$ y $\mathrm{Tf}$ se mantuvieron estables hasta los 12 meses. $\mathrm{Si}$ bien se observa una repoblación de $T d$ al año post tratamiento, las bolsas periodontales mantuvieron los valores de PS logrados con la terapia.

\section{Resultado del Tratamiento}

El análisis de los resultados de nuestra experiencia acorde a los porcentajes de éxito de tratamiento según los criterios clínicos propuestos en la escala de Lundgren ${ }^{(29)}$ se observan en la Tabla 4.

Al correlacionar los hallazgos clínicos con la identificación bacteriológica, observamos que los sitios caracterizados como de nivel 1 de "éxito" terapéutico se correspondieron con una menor detección de las bacterias estudiadas.

El $63 \%$ de los sitios evaluados a los 3 meses y el $67 \%$ a los 12 meses, no presentaban ninguna de las cinco especies estudiadas.

Tabla 1. Criterios de inclusión para los distintos niveles de "éxito".

Nivel 1
a. Profundidad de sondaje $\leq 4 \mathrm{~mm}$.
b. Ausencia de sangrado al sondaje.
c. Sin aumento en la pérdida de inserción clínica.
Nivel 2
b. Ausencia de sangrado al sondaje.
c. Sin aumento en la pérdida de inserción clínica.
Nivel 3
c. Sin aumento en la pérdida de inserción clínica.

Tabla 2. Datos clínicos en el inicio del tratamiento y a los 7 días, 3 y 12 meses post terapia.

\begin{tabular}{|l|l|l|l|l|}
\hline & INICIO & 7 DIAS & 3 MESES & 12 MESES \\
\hline Placa bacteriana & $44(100)$ & $15(34.1)^{\star}$ & $23(52.3)^{\star}$ & $27(67.4)^{\star}$ \\
\hline Hemorragia & $44(100)$ & $21(47.7)^{\star}$ & $19(43.2)^{\star}$ & $16(37.2)^{\star}$ \\
\hline Supuración & $5(11.3)$ & $1(2.3) \dagger$ & $1(2.3) \dagger$ & $3(7)$ \\
\hline Profundidad al sondaje $(\mathrm{mm})$ & $5.91 \pm 1.16$ & $4.39 \pm 1.26^{*}$ & $3.68 \pm 1.16^{*}$ & $3.91 \pm 1.49^{\star}$ \\
\hline Nivel de Inserción Clínica $(\mathrm{mm})$ & $6.54 \pm 2.02$ & $5.89 \pm 1.65^{*}$ & $5.39 \pm 2.05^{*}$ & $5.16 \pm 2.32^{\star}$ \\
\hline
\end{tabular}

Los números de Placa bacteriana, Hemorragia y Supuración expresan el número de sitios positivos y entre paréntesis el porcentaje de sitios positivos para esa variable. Profundidad de Sondaje y Nivel de Inserción Clínica representan media $\pm S D * 0<0.0001+p=0.018$ vs. Inicio

Tabla 3. Porcentaje de sitios positivos para cada una de las especies bacterianas estudiadas en cada uno de los momentos del estudio.

\begin{tabular}{|l|l|l|l|l|}
\hline & I (n=44) & 1S (n=44) & 3M (n=44) & 12M (n=43) \\
\hline Pg & $29(65.9 \%)$ & $20(45.45 \%)^{*}$ & $15(34.09 \%) \dagger$ & $13(30.23 \%)^{* *}$ \\
Tf & $24(54.54 \%)$ & $11(25 \%) \dagger \dagger$ & $6(13.63 \%)^{* *}$ & $9(20.93 \%) \xi$ \\
Td & $18(40.90 \%)$ & $5(11.36 \%)^{*}$ & $4(9.09 \%) \dagger$ & $10(23.25 \%)$ \\
Aa & $4(9.09 \%)$ & $1(2.27 \%)$ & $5(11.36 \%)$ & $4(9.3 \%)$ \\
Pi & $3(6.81 \%)$ & $2(4.54 \%)$ & $2(4.54 \%)$ & $1(2.27 \%)$ \\
\hline
\end{tabular}

$\mathrm{I}=$ inicio del tratamiento, $1 \mathrm{~S}=$ una semana post tratamiento, $3 \mathrm{M}=$ tres meses post tratamiento, $12 \mathrm{M}=$ doce meses post tratamiento. Los números representan el número de sitios positivos en los que se identificó cada bacteria y entre paréntesis el porcentaje de sitios: ${ }^{*} p=0.002 ; \uparrow p=0.006 ;{ }^{* *} p<0.001$; $\dagger+p=0.022 ; \xi p=0.001$ vs. Inicio.

Tabla 4. Frecuencia de sitios positivos para cada bacteria sola o combinada en cada fase del estudio asociado a la Profundidad de Sondaje Media.

\begin{tabular}{|l|c|c|c|c|c|c|c|c|}
\hline & \multicolumn{2}{|c|}{ I } & \multicolumn{2}{c|}{ 1S } & \multicolumn{2}{c|}{$3 \mathrm{M}$} & \multicolumn{2}{c|}{$12 \mathrm{M}$} \\
\cline { 2 - 10 } & $\begin{array}{c}\text { Sitios } \\
\text { positivos }\end{array}$ & $\begin{array}{c}\text { PS } \\
\text { media }\end{array}$ & $\begin{array}{c}\text { Sitios } \\
\text { positivos }\end{array}$ & $\begin{array}{c}\text { PS } \\
\text { media }\end{array}$ & $\begin{array}{c}\text { Sitios } \\
\text { positivos }\end{array}$ & $\begin{array}{c}\text { PS } \\
\text { media }\end{array}$ & $\begin{array}{c}\text { Sitios } \\
\text { positivos }\end{array}$ & $\begin{array}{c}\text { PS } \\
\text { media }\end{array}$ \\
\hline$P g+T f$ & $9(20 \%)$ & 6.6 & $5(11 \%)$ & 4.8 & $2(4 \%) \dagger$ & 4 & $2(4 \%)+\dagger$ & 7 \\
\hline$T f+T d$ & $4(9 \%)$ & 6.8 & 0 & - & 0 & - & $1(2 \%)$ & 7 \\
\hline$P g+T d$ & $4(9 \%)$ & 6,5 & $1(2 \%)$ & 3 & $1(2 \%)$ & 6 & $2(4 \%)$ & 2 \\
\hline$P g+T f+T d$ & $3(7 \%)$ & 5 & $3(7 \%)$ & 5 & $2(4 \%)$ & 3 & $3(7 \%)$ & 5 \\
\hline$P g+T d+A a$ & $1(2 \%)$ & 6 & 0 & - & 0 & - & $1(2 \%)$ & 3 \\
\hline$P g+T d+T f+A a$ & $2(4 \%)$ & 6 & 0 & - & 0 & - & 0 & - \\
\hline$P g+T d+T f+P i$ & $2(4 \%)$ & 5 & 0 & - & 0 & - & $1(2 \%)$ & 4 \\
\hline$T f+T d+P i$ & $1(2 \%)$ & 7 & 0 & - & 0 & - & 0 & - \\
\hline$P g+A a$ & $1(2 \%)$ & 5 & 0 & - & $2(4 \%)$ & 3.5 & 0 & - \\
\hline$P g+P i$ & 0 & - & $1(2 \%)$ & 4 & $2(4 \%)$ & 2 & 0 & - \\
\hline$P g$ & $7(16 \%)$ & 5.42 & $10(23 \%)$ & 4.7 & $6(14 \%)$ & 3.5 & $4(9 \%)$ & 4 \\
\hline$T f$ & $3(7 \%)$ & 5.33 & $3(7 \%)$ & 4 & $2(4 \%)$ & 4 & $2(4 \%)$ & 4 \\
\hline$T d$ & $1(2 \%)$ & 5 & $1(2 \%)$ & 4 & $1(2 \%)$ & 7 & $2(4 \%)$ & 4 \\
\hline$A a$ & 0 & - & $1(2 \%)$ & 4 & $3(7 \%)$ & 4.66 & $3(7 \%)$ & 3 \\
\hline$P i$ & 0 & - & $1(2 \%)$ & 4 & 0 & - & 0 & - \\
\hline Otras & $6(14 \%)$ & 5.83 & $18(41 \%)^{* *}$ & 4.27 & $23(52 \%)^{*}$ & 3.52 & $22(50 \%)^{*}$ & 3.68 \\
\hline
\end{tabular}

Otras= sitios positivos para bacterias Gram negativas que no se corresponden con $\mathrm{Aa}, \mathrm{Pg}, \mathrm{Pi}, \mathrm{Td}$ y Tf $\mathrm{I}=$ inicio del tratamiento, $1 \mathrm{~S}=$ una semana post tratamiento, $\cdot 3 \mathrm{M}=$ tres meses post tratamiento, $12 \mathrm{M}=$ doce meses post tratamiento. PS= profundidad de sondaje Los números representan el número de sitios positivos en los que se identificó cada bacteria o las combinaciones entre ellas y entre paréntesis el porcentaje de sitios positivos. PS= se expresa como la media obtenida de los sitios positivos para cada bacteria o grupo de bacterias. $\dagger p=0.039 ; \dagger+p=0.016 ;{ }^{*} p<0.0001 ;{ }^{* *} p<0.008$ vs. Inicio.

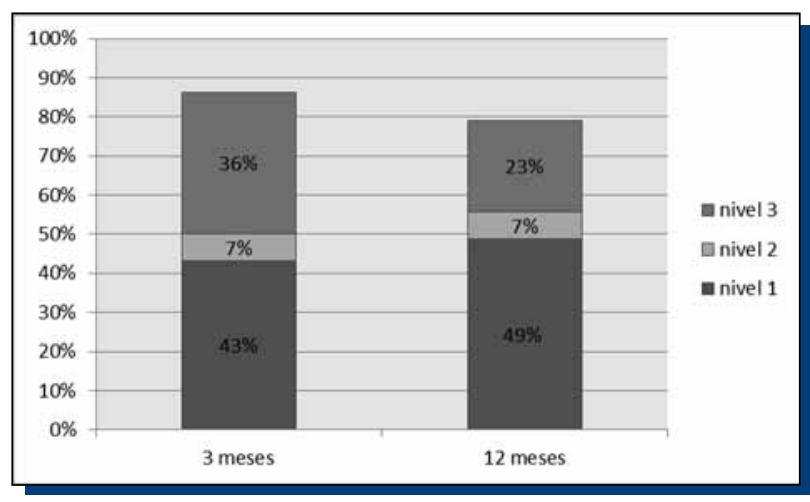

Gráfico 1. Frecuencia acumulada de sitios clínicamente "sanos" a los 3 y 12 meses post tratamiento, de acuerdo a los niveles 1 a 3 de éxito de Lundgren. 


\section{DISCUSIÓN}

En el presente estudio se evaluaron sitios con PS $\geq 5 \mathrm{~mm}$, con el propósito de analizar el efecto de la terapia mecánica periodontal no quirúrgica sobre la composición microbiana y los parámetros clínicos. Hasta el momento no se conocen en Argentina estudios a 12 meses de seguimiento que evalúen la composición bacteriana antes y después del tratamiento periodontal.

Según nuestras observaciones, antes del tratamiento la prevalencia fue para $\mathrm{Pg}$ del $66 \%, \mathrm{Tf}$ del $55 \%$ y para $\mathrm{Td} 41 \%$, mientras que $A a$ se identificó en el $9 \%$ de los sitios. Muchos autores describen a Aa con una importante participación en la destrucción de los tejidos periodontales, especialmente si está asociada a otras especies ${ }^{(30)}$, sin embargo, Johansson y cols. ${ }^{(31)}$ demostraron que $P g$ es capaz de inhibir la actividad de las leucotoxinas de $A a$. En nuestro estudio $P g$ fue la especie más frecuentemente identificada. La observación de Johansson podría explicar por qué los sitios identificados con $A a$ no corresponden a sitios de progresión.

La especie bacteriana que mayor diferencia presenta con los resultados de otros estudios fue $P$ intermedia que se detectó en el $7 \%$ de los sitios, mientras que otros autores ${ }^{(11,27,32)}$ la identifican entre 43 y $90 \%$. Los estudios de complejos microbianos ${ }^{(33)}$ sugieren además que, $\mathrm{Pi}$, como otras bacterias del complejo naranja, precedería a las del complejo rojo $(B f, T d$ y $P g)$. En general, en nuestra población esta bacteria es descrita en baja frecuencia ${ }^{(10)}$.

En cuanto a las asociaciones bacterianas, hallamos, antes del tratamiento, que el mayor porcentaje de sitios se encontraba poblado por grupos de 2 especies y que estos sitios mostraban las mayores profundidades de sondaje. En este momento del estudio también se encontraron grupos de tres y cuatro especies bacterianas. Ready y cols. ${ }^{(34)}$, describieron mayor severidad en lesiones pobladas por sólo una especie $(\mathrm{Pg}, \mathrm{Tf}$ ○ $\mathrm{Aa})$ si se comparaba con las que contenían asociaciones de dos o tres de ellas, a diferencia de las conclusiones de Chen ${ }^{(30)}$ que encontró mayor gravedad en las lesiones habitadas por las tres especies involucradas en su estudio $(P g, A a$ y $T d)$ que en las que se hallaban una o dos de ellas.

La reducción en la prevalencia de las bacterias del complejo rojo ( $B f, T d$ y $P g)$ se evidenció en el primer control (a una semana) de implementado el tratamiento, si bien no en la magnitud que registran otros autores ${ }^{(35)}$. Rhemrev y cols. ${ }^{(36)}$ reportaron una reducción significativa de $P g, P i, M$ micros y $F$ nucleatum a 2 semanas, mientras que $A a$ y $C$ rectus se mantuvieron sin cambios y la reducción de $T f$ fue significativa sólo a una semana de implementada la terapia básica. $P g$ y $T f$ fueron reducidas a 1 mes con raspado y alisado, pero no $A a^{(37)}$. Darby ${ }^{(27)}$ demostró disminución significativa de los porcentajes de sitios positivos para $\mathrm{Pi}, T f$ y $T d$ en 28 pacientes con periodontitis crónica, a 8 meses de finalizado el raspado y alisado.

En el presente estudio, la reducción de sitios infectados fue más evidente a los 3 meses post tratamiento, coincidiendo con publicaciones de Haffajee ${ }^{(19)}$, y Derdilopoulou ${ }^{(32)}$. Este autor comparó el efecto de cuatro modalidades de raspado y alisado sobre la microbiología de bolsas periodontales y hallaron que a 3 meses de aplicado el tratamiento, $T d, P g, P i, T f$ y $A$ a disminuyeron su prevalencia significativamente, sin embargo, 6 meses después de finalizada la terapia activa, los porcentajes de sitios positivos para las cinco especies bacterianas aumentaron nuevamente, alcanzando valores similares a los de pre-tratamiento.

Si bien el número de sitios libres de $P g$ y $T f$ se mantuvo estable hasta los 12 meses, resulta llamativa la repoblación de sitios por $T d$, especie que aumentó su prevalencia, aunque sin alcanzar los valores iniciales. La repoblación depende de la severidad de la lesión, la efectividad del desbridamiento y principalmente de la presencia de reservorios para estos microorganismos en sitios inaccesibles para el raspado y alisado como amígdalas, lengua y mucosas, además algunos microorganismos pueden invadir los tejidos blandos, las irregularidades de la superficie radicular y los túbulos dentinarios ${ }^{(21)}$. Particularmente $T d$ ha demostrado recurrencia significativa entre 3 y 6 meses posttratamiento aun cuando éste incluía, además del raspado y alisado, antibióticos y buches con clorhexidina. Esta recurrencia se atribuye a su presencia en localizaciones extracreviculares ${ }^{(38)}$.

Los datos clínicos relevados indicarían que la terapia básica periodontal resulta efectiva en términos de reducción de la profundidad de sondaje, mantenimiento y/o ganancia en el nivel de inserción clínica, como así también disminución del porcentaje de sitios con placa y hemorragia al sondaje a 3 meses de realizado. Estos resultados concuerdan con otros estudios que describen los efectos clínicos del raspado y alisado radicular en similar período de tiempo ${ }^{(14,21,24,27,39,40)}$. Se observó un leve aumento, aunque no significativo, a los 12 meses, en la presencia de PB. Esto reflejaría una disminución en el compromiso de los pacientes, tal vez debido al tiempo transcurrido sin refuerzo en la motivación.

Se han publicado numerosos trabajos que evalúan la efectividad de la terapia básica periodontal, la mayoría de ellas se basan en la variación de la media de los parámetros clínicos, resultando así tratamientos más o menos exitosos. Sin embargo, pocos o ninguno demuestran si esas variaciones representan parámetros de éxito clínico, en cuanto a recuperación de un estado periodontal compatible con salud. La aplicación de los diferentes niveles de éxito terapéutico y la correlación con la composición bacteriana en cada uno de los niveles nos ofrece una idea más aproximada del efecto que la terapia mecánica no quirúrgica produciría en los sitios estudiados.

Los resultados de la presente investigación demostrarían que la terapia mecánica periodontal aplicada a bolsas profundas de dientes unirradiculares, es capaz de producir dos efectos: un cambio en la composición bacteriana de las bolsas y una modificación en el hábitat debida principalmente a la disminución de los signos inflamatorios. El hábitat, por su parte, condiciona la repoblación, si una especie depende de la presencia de bolsas profundas o productos resultantes de la inflamación, entonces esas especies existirán en reducido número o serán eliminadas de un sitio periodontalmente tratado con éxito.

Por ser un estudio a largo plazo, con un elevado índice de abandono por parte de los pacientes, los resultados del presente trabajo se hallan limitados al número reducido de muestras de los pacientes que completaron el tratamiento.

El estudio en una muestra mayor arrojaría resultados que nos ayudarían a comprender un modelo de repoblamiento bacteriano en bolsas periodontales no recuperadas, además de conocer la participación de factores de riesgo en la evolución post tratamiento de parámetros periodontales.

El objetivo del trabajo fue identificar cinco especies bacterianas de probado vínculo con las enfermedades periodontales, por medio de PCR antes y después del tratamiento. Si bien con PCR se detecta presencia/ausencia de DNA bacteriano, éste estaría representando un microorganismo que en algún momento estuvo activo en la profundidad de la bolsa y en consecuencia, estaría relacionado con la destrucción del aparato de inserción periodontal. La factibilidad de encontrar falsos positivos y falsos negativos entre los resultados, se reduce con la incorporación de tubos blancos sin DNA bacteriano, tubos con DNA bacteriano de bacterias proveniente de cultivo y tubos duplicados de cada muestra.

En diversos estudios ${ }^{(12,41-43)}$ se ha demostrado que la aplicación de un programa regular de mantenimiento periodontal (terapia periodontal de soporte), reduce significativamente la pérdida de inserción y la pérdida de piezas dentarias a largo plazo, a la vez que mejora el control de placa bacteriana y reduce la inflamación. El intervalo óptimo de tiempo para realizar las citas de mantenimiento suele decidirse empíricamente.

En el control a 3 meses, se observó que los sitios con parámetros clínicos de salud periodontal, se encontraban, en su mayoría libres de las especies patógenas estudiadas. De esta observación se infiere que el estudio microbiológico de las bolsas en este momento podría servir como indicador de riesgo a fin de establecer el intervalo para las citas de mantenimiento o la planificación de terapia periodontal complementaria.

Por otra parte, seria interesante conocer el comportamiento de la recolonización en sitios que no alcanzaron la salud periodontal asociado con terapias complementarias como el uso de antibióticos.

\section{DECLARACIÓN DE INTERÉS}

Los autores declaran no tener conflictos de interés. 


\section{REFERENCIAS BIBLIOGRÁFICAS}

1. International workshop for a classification of periodontal diseases and conditions Consensus report: Chronic periodontitis. Ann Periodontol, 1999, 4(1): 38. 2. Renvert S, Persson R. Supportive periodontal therapy. Periodontology 2000, 2004; 36: 179-195.

3. Mendoza C, Arteaga O, Gamonal J. Investigación epidemiológica en enfermedad periodontal en América Latina. Rev Chil Period Oseoint, 2006; 3: 7-13.

4. Holt S, Ebersole J. Porphyromonas gingivalis, Treponema denticola and Tannerella forsythia: The "red complex", a prototype polybacterial pathogenic consortium in periodontitis. Periodontology 2000, 2005; 38: 72-122.

5. Umeda M, Chen C, Bakker I, Contreras A, Morrison JL, Slots J. Risk indicators for harboring periodontal pathogens. J Periodontol, 1998; 69(10): 1111-1118.

6. Sanz M, Van Winkelhoff A, Herrera D, Delleminj-Kippuw N, Simón R, Winkel E. Differences in the composition of the subgingival microbiota off two periodontitis populations of different geographical origin. A comparison between Spain and the Netherlands. Eur J Oral Sci, 2000; 108(5): 383-392.

7. Haffajee AD, Bogren A, Hasturk H, Feres M, Lopez NJ, Socransky SS. Subgingival microbiota of chronic periodontitis subjects from different geographic locations. $J$ Clin Periodontol, 2004; 31: 996-1002.

8. Teles R, Haffajee A, Socransky S. Microbiological goals of periodontal therapy. Periodontology 2000, 2006; 42: 180-218.

9. Herrera D, Contreras A, Gamonal J, Oteo A, Jaramillo A, Silva N, Sanz M, Botero JE, León R. Subgingival microbial profiles in chronic periodontitis patients from Chile, Colombia and Spain. J Clin Periodontol, 2008; 35(2): 106-113.

10. Parodi R, Moreno Barral J, Sembaj A, Bazzano G, Usin M, Tabares S. PCR for the detection and tipification of periodontal pathogens. J Dent Res, 2001; 80(4): 960 11. Ashimoto A, Chen C, Bakker I, Slots J. Polymerase chain reaction detection of 8 putative periodontal pathogens in subgingival plaque of gingivitis and advanced periodontal lesions. Oral Microbiol Inmunol, 1996; 11: 266-273.

12. Shiloah J, Patters M. Repopulation of periodontal pockets by microbial pathogens in the absence of supportive therapy. J Periodontol, 1996; 67: 130-139. 13. Estrela CR, Pimenta FC, Alencar AH, Ruiz LF, Estrela C. Detection of selected bacterial species in intraoral sites of patients with chronic periodontitis using multiplex polymerase chain reaction. J Appl Oral Sci, 2010; 18(4): 426-431.

14. Suvan J. Effectiveness of mechanical nonsurgical pocket therapy. Periodontology 2000, 2005; 37: 48-71.

15. Cugini MA, Haffajee AD, Smith C, Kent Jr RL, Socransky SS. The effect of scaling and root planing on the clinical and microbiological parameters in periodontal diseases: 12-month results. J Clin Periodontol, 2000; 27: 30-36.

16. Williams RC. Periodontal disease: The emergence of a new paradigm. Compendium of Continuing Education in Dentistry, 1998; 19: 5-9.

17. Drisko CH. Non surgical periodontal therapy. Periodontology 2000, 2001; 25 : 77-88.

18. Faveri M, Gursky LC, Feres M, Shibli JA, Salvador SL, de Fiqueiredo LC Scaling and root planing and clorhexidine mouthrinses in the treatment of chronic periodontitis: A randomized, placebo-controlled clinical trial. J Clin Periodontol, 2006; 33: 819-828

19. Haffajee A, Teles $R$, Socransky $S$. The effect of periodontal therapy on the composition of the subgingival microbiota. Periodontology 2000, 2006; 42: 219-248. 20. Lowenguth $R$, Greenstein $G$. Clinical and microbiological response to non surgical mechanical periodontal therapy. Periodontology 2000, 1995; 9: 14-22.

21. Colombo A, Teles R, Torres $M$, Rosalém W, Mendes $M$, Souto R, Uzeda $M$. Effects of non surgical mechanical theraphy on the subgingival microbiota of brazilians with untreated chronic periodontits: 9-month results. J Periodontol, 2005 76: $778-784$

22. Caffesse R, Sweeney PL, Smith BA. Scaling and root planning with and without periodontal flap surgery. J Clin Periodontol, 1986; 13: 205-210.

23. Chan YK, Needleman IG, Clifford LR. Comparison of four methods of assessing root surface debridement. J Periodontol, 2000; 71: 385-393.
24. Haffajee A, Cugini M, Dibart S, Smith C, Kent R Jr., Socransky S. The effect of SRP on the clinical and microbiological parameters of periodontal diseases. $J$ Clin Periodontol, 1997; 24(5): 324-334.

25. Cobb CM. Microbes, inflammation, scaling and root planing, and the periodontal condition. J Dent Hyg, 2008; 82 Suppl 3: 4-9.

26. Tanner A, Paster BJ, Lu SC, Kanasi E, Kent Jr. R, Van Dyke T, Sonis T. Subgingival and tongue microbiota during early periodontitis. J Dent Res, 2006 85(4): 318-323.

27. Darby IB, Hodge PJ, Riggio MP, Kinane DF. Clinical and microbiological effect of scaling and root planning in smoker and non-smoker chronic and aggressive periodontits patients. J Clin Periodontol, 2005; 32: 200-206.

28. Consensus report: Section of epidemiology. Ann Periodontol, 1996; 1: 216-218. 29. Lundgren D, Asklöw B, Thorstensson H, Härefeldt AM. Success rate in periodontal treatment as related to choice of evaluation criteria. Presentation of an evaluation criteria staircase for cost-benefit use. J Clin Periodontol, 2001; 28: 23-30. 30. Chen LL, Wu YM, Yan J, Sun WL, Sun YZ, Ojcius D. Association between coinfection of Porphyromonas gingivalis, Actinobacillus actynomicetemcomitans and Treponema denticola and periodontal tissue destruction in chronic periodontitis. Chin Med J (Engl), 2005; 118(11): 915-921.

31. Johansson A, Hanstrom L, Kalfas S. Inhibition of Actinobacillus actinomycetemcomitans leukotoxity by bacteria from subgingival flora. Oral Microbial Inmunol, 2000; 15: 218-225.

32. Derdilopoulou FV, Nonhoff J, Neumann K, Kielbassa AM. Microbiologica findings after periodontal therapy using curettes. Er: YAG laser, sonic and ultrasonic scalers. J Clin Periodontol, 2007; 34: 588-598.

33. Socransky SS, Haffajee AD, Cuggini MA, Smith C, Kent Jr. RL. Microbial complexes in subgingival plaque. J Clin Periodontol, 1998; 25: 134-144.

34. Ready D, D'Aiuto F, Spratt DA, Suvan MS, Tonetti MS, Wilson M. Disease severity associated with presence in subgingival plaque of Porphyromonas gingivalis, Aggregatibacter actinomycetemcomitans and Tannerella forsythia, singly or in combination, as detected by nested multiplex PCR. J Clin Microbiol, 2008 46(10): 3380-3383.

35. Segelnick S, Weinberg M. Reevaluation of initial therapy. When is the appropriate time? J Periodontol, 2006, 77: 1598-1601.

36. Rhemrev GE, Timmerman MF, Veldkamp I, Van Winkelhoff AJ, Van der Velden $U$. Inmediate effect of instrumentation on the subgingival microflora in deep inflamed pockets under strict plaque control. J Clin Periodontol, 2006; 33: 42-48.

37. Takamatsu N, Yano K, He T, Umeda M, Ishikawa I. Effect of initial periodontal therapy on the frequency of detecting Bacteroides forsythus, Porphyromonas gingivalis and Actinobacillus actinomycetemcomitans. J Periodontol, 1999, 70: 574 580

38. Johnson JD, Chen R, Lenton PA, Zhang G, Hinrichs JE, Rudney JE. Persistance of extracrevicular bacterial reservoirs after treatment of aggressive periodontitis. $J$ Periodontol, 2008; 79: 2305-2312.

39. Haffajee A, Torresyap G, Socransky SS. Clinical changes following four different periodontal therapies for the treatment of chronic periodontitis: 1 year results. J Clin Periodontol, 2007; 34: 243-253.

40. Yang $\mathrm{H}$, Xu Y, Ding $\mathrm{Y}$, Zhoo $\mathrm{H}$. Distribution of five periodontal pathogens in subgingival plaque in chronic periodontitis. West China Journal of Stomatology, 2007; 25: 470-473.

41. Axelsson $P$, Lindhe J. The significance of maintenance care in the treatment of periodontal disease. J Clin Periodontol, 1981; 8(4): 281-294.

42. American Academy of Periodontology. Position paper. Periodontal maintenance. J Periodontol, 2003; 74: 1395-1401.

43. Gaunt F, Devine M, Pennington M, Vernazza C, Gwynnett E, Steen N, Heasman $P$. The cost-effectiveness of supportive periodontal care for patients with chronic pèriodontitis. J Clin Periodontol, 2008; 35(Suppl. 8): 67-82. 